\title{
In Situ Diagnosis of a Gear-Tooth Surface Damage Using Laser Scattering*
}

\author{
Eiichirou TANAKA**, Kazuteru NAGAMURA***, Kiyotaka IKEJO*** \\ and Ryozo NEMOTO**** \\ **Shibaura Institute of Technology, \\ 307 Fukasaku, Minuma-ku, Saitama, 337-8570 Japan \\ E-mail: tanakae@sic.shibaura-it.ac.jp \\ ***Hiroshima University, \\ 1-4-1 Kagamiyama, Higashi-hiroshima, 739-8527 Japan \\ **** Tokyo Metropolitan College of Industrial Technology, \\ 8-17-1 Minami-senju, Arakawa-ku, Tokyo, 117-8523 Japan
}

\begin{abstract}
We propose herein an in situ method to remotely diagnose gear-tooth damage using scattering of a laser beam. The proposed method provides early and accurate diagnosis of the gear-tooth-surface condition. A tooth surface is first irradiated at oblique incidence by a zone-covering laser beam, and the zone is scanned along the surface of the gear tooth by the rotation of the gear. By analyzing variations in laser scattering between benchmark data and the current data, we can estimate the condition of the gear-tooth surface in terms of abnormal abrasion, pitting, spalling, etc. To test the method, we used it to remotely detect gear-teeth pitting in an experiment during which we simultaneously measured the vibration and sound of the gearbox and pedestal. Our analysis shows that the laser-scattering measurements reveal pitting more clearly and at an earlier stage than does the vibration and noise measurements. Therefore, we conclude that the proposed method estimates the tooth-surface condition with sufficient accuracy to assess the lifetime of the gear. Furthermore, we used the proposed method to diagnose real gear-teeth in situ in practical gearboxes to verify that the method yields an accurate diagnosis of the tooth surface of a lubricated gear. We found that by attaching a cover to the laser receiver; the remote measurements were unaffected by the choice or method of lubrication. For force-feed lubrication, pitting was detected for every speed range under $1800 \mathrm{rpm}$. These results demonstrate that the proposed method can diagnose the tooth surface of lubricated gears in practical gearboxes. Finally, we developed an automatic damage diagnosis method that is capable of detecting pitting by analyzing the laser-scattering signal from damaged teeth combined with that from the same teeth prior to the damage.
\end{abstract}

Key words: Gear, Damage Diagnosis, Laser Beam, Pitting, Lubricant

\section{Introduction}

Much research effort has been focused on studying industrial machines with gears from various viewpoints, such as efficiency, vibration, and noise. In recent years, the needs of machine maintenance have led to demands of remote diagnostic techniques for gear-teeth. Different maintenance methods have been developed to diagnose damage to the gear-tooth surface using different techniques involving, for example, acoustic emission ${ }^{(1)}$, sound ${ }^{(2)}$, tooth root $\operatorname{strain}^{(3)}$ and vibration. ${ }^{(3)(4)}$ However, for early detection of gear-tooth abnormalities, such diagnostic methods are not optimum. And if the damaged tooth can be

2011 (No. 11-0302) [DOI: 10.1299/jamdsm.5.199]

Copyright (C) 2011 by JSME 
detected, it is difficult to grasp the damaged area on the tooth surface in detail. To address this problem, we developed a method that uses a laser beam to perform a remote diagnosis ${ }^{(5)}$ and verified that it accurately diagnoses gear-tooth-surface abnormalities of a lubricated gear in a practical gearbox. We also developed real-time pit-detection algorithm with the objective of creating an automated gear-tooth diagnosis system.

\section{Nomenclature}

$m$ : Module [mm]

$z$ : Number of teeth

$\alpha$ : Pressure angle [rad]

$r$ : Radius of the circle which is able to define the laser beam as the tangential line [mm]

$r_{0}$ : Radius of the pitch circle, $r_{0}=m z / 2[\mathrm{~mm}]$

$r_{a}$ : Radius of the tip circle, $r_{a}=m z / 2+m[\mathrm{~mm}]$

$r_{b}$ : Radius of the base circle, $r_{b}=m z(\cos \alpha) / 2[\mathrm{~mm}]$

\section{Laser-scattering damage diagnosis method}

Our method can be explained using Fig. 1. First, a gear-tooth surface is irradiated at an oblique angle by a zone-covering laser beam, as shown in Fig. 1(a). Owing to the rotation of the gear, the irradiated zone is scanned over the tooth's surface, as shown in Fig. 1(b). The laser reflects both specularly and diffusely from the gear-tooth surface, and as the pitting on the surface increases, the diffuse reflection (i.e., scattering) increases at the expense of the specular reflection. The optical detector detects a portion of the scattered laser light and converts it into a proportional voltage, which is read out in real time (i.e., as the gear is rotating) and from which the location of the damage on the tooth surface may be derived from the angle of the rotating gear.

Consider the angle $\theta$, which is the angle through which the gear rotates to reach to an arbitrary point $x$ on the gear tooth from the point where the laser beam irradiates the base circle (see Fig. 2). This angle may be defined as

$$
\theta=\operatorname{inv}\left(\cos ^{-1}\left(r_{\mathrm{b}} / R\right)\right)+\cos ^{-1}(r / R)-\cos ^{-1}\left(r / r_{\mathrm{b}}\right) .
$$

When a damaged point on a gear-tooth surface is detected, $\theta$ for this point can be determined from the laser-scattering data. Once $\theta$ is known, $R$ can be calculated from Eq. (1). By using the result of $R$, the damaged point on the gear-tooth surface can be expressed as a distance $x$ from the base circle:

$$
x=r_{b} \tan ^{2}\left(\cos ^{-1}\left(r_{b} / R\right)\right) / 2 .
$$

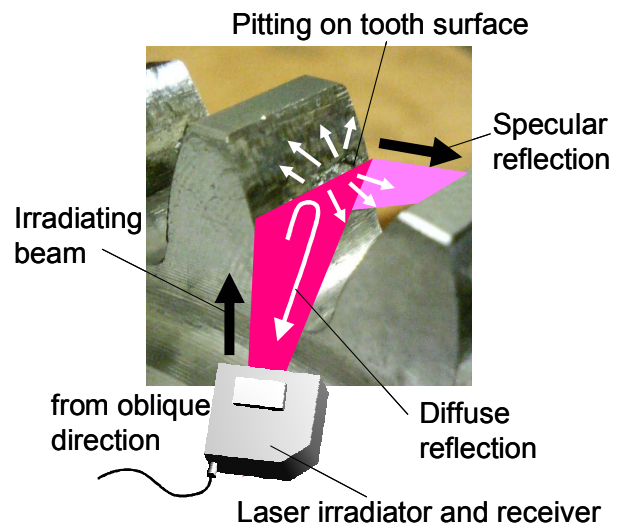

(a) Tooth width direction

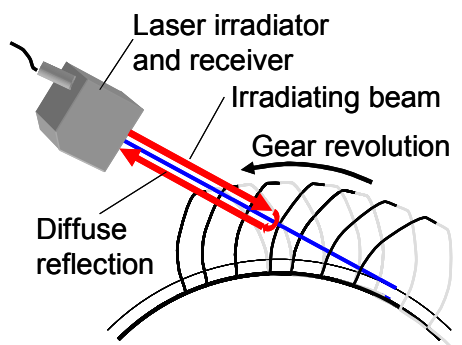

(b) Tooth profile direction

Fig. 1 Principle for measuring gear-tooth surface by laser reflection 


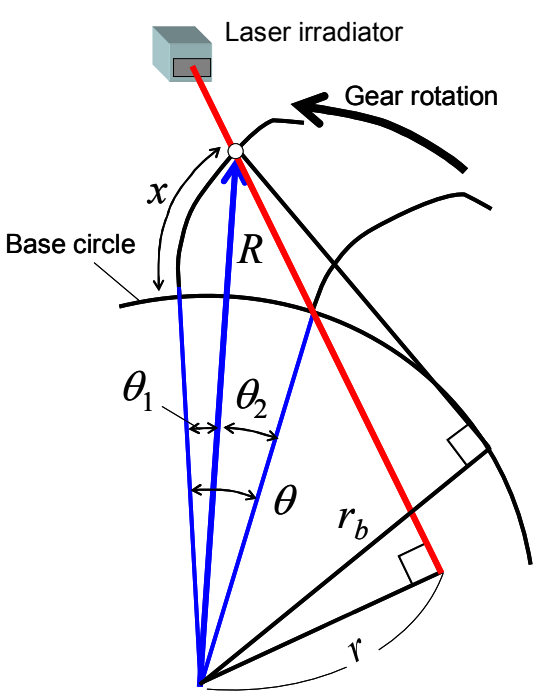

Fig. 2 Geometry connecting the angles between laser beam and the damaged point on gear-tooth-surface

By comparing the initial and current laser-scattering data, we can estimate the tooth-surface conditions and detect anomalies such as initial or abnormal abrasion, pitting, and spalling.

Therefore, the variations of laser reflection between the initial and the present conditions can be compared, and we can estimate conditions on the tooth surface such as initial or abnormal abrasion, pitting, spalling, etc. from these results.

\section{Pitting Experiment}

To test the validity of the proposed method, we performed a pitting experiment with a practical gearbox. During the experiment, the laser scattering, gear vibration, adjacent noise, and accumulated wear were measured at each load repetition number $N$ (in cycles). To obtain results as a factor of laser scattering, test gears were detached from the gearbox to measure the initial laser scattering. A power-circulating-type gear testing machine was used for the pitting experiment. Dimensions of the test gear pair are given in Table 1. The conditions of the pitting experiment are given in Table 2.

Figure 3 shows the result of the accumulated wear as a function of the load repetition number $N$. The results show that wear increases above $N=3.5 \times 10^{5}$, and it shows the occurrence and progression of pitting. Figure 4 shows photos of the condition of the driving gear tooth surface corresponding to various load repetition numbers $N$. At $N=0$ for tooth 15 [Fig. 4(a)], only cutting scars by hobbing appear on the tooth surface. When the load repetition number increases beyond $N=1 \times 10^{5}$, the tooth-surface roughness becomes less apparent, and the photo at $N=2 \times 10^{5}$ shows a smoother tooth surface than that seen at $N=$ $1 \times 10^{5}$. However, for tooth 29, pitting is evident in the photo at $N=2 \times 10^{5}$. At $N=4 \times 10^{5}$, every tooth generated pitting and pitting increased as the logarithm of load repetition number above $N=4 \times 10^{5}$.

Figure 5 shows laser-scattering data for the tooth surface of the driving gear as a function of gear-rotation angle. By comparing these data with those of the baseline experiment, we can understand the influence of variations in irradiation angle and distance. For tooth 15 , the voltage at $N=2 \times 10^{5}$ was the lowest because the tooth surface scattered the least. For tooth 29, the voltage at $N=2 \times 10^{5}$ increased around the pitch point in comparison with the results at $N=1 \times 10^{5}$. Therefore, an area of pitting can be inferred from these data. After $N=4 \times 10^{5}$, the area of increased scattering expanded monotonically with $N$, signaling a further increase in pitting. 
Table 1 Dimensions of test gear pair (driving and driven gears)

Gear type

Module $m[\mathrm{~mm}]$

Pressure angle $\alpha$ [deg]

Number of teeth $z_{1} / z_{2}$

Pitch circle diameter $[\mathrm{mm}]$

Face width [mm]

Finishing method of the gear

Grade (JIS B 1702-1, JIS B 1702-2(1998))

Material

Heat treatment

Base tangent length $E_{n}[\mathrm{~mm}]$
Spur gear

4

20

$29 / 29$

116

10

Hobbed

4

$\mathrm{S} 45 \mathrm{C}$

Thermal refining

42.805 (3 tooth)

Table 2 Conditions of the pitting experiment

\begin{tabular}{l|c}
\hline \hline Input rotational speed $[\mathrm{rpm}]$ & 1800 \\
Input torque $[\mathrm{N} \cdot \mathrm{m}]$ & 145 \\
Lubricating oil & Turbine oil (ISO VG32) \\
Oil temperature $[\mathrm{K}]$ & $333 \pm 2$ \\
\hline
\end{tabular}

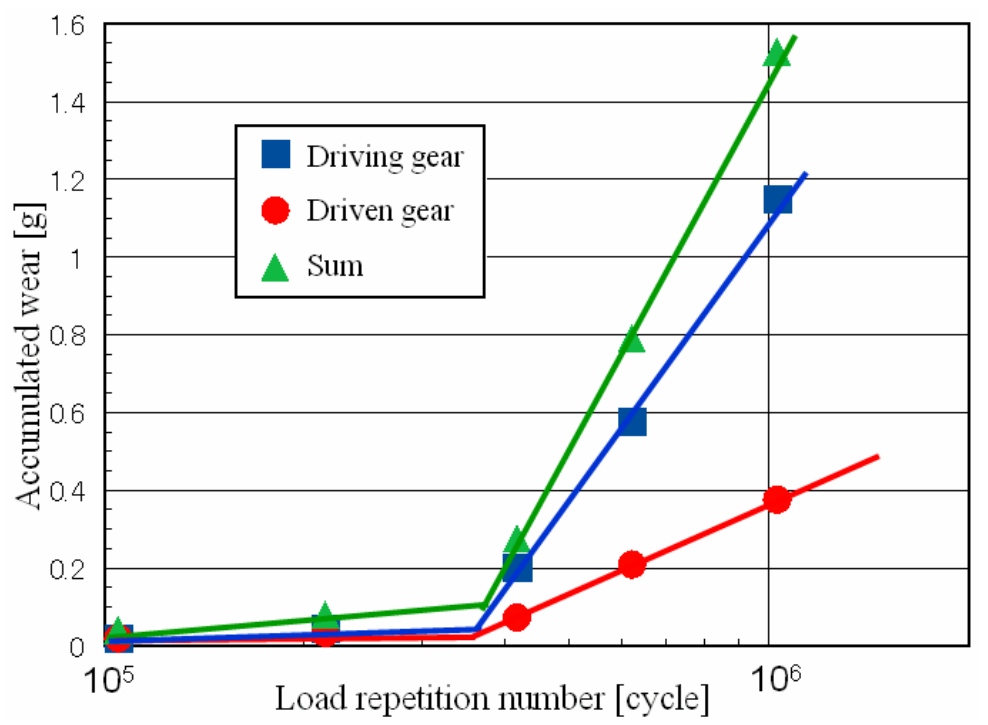

Fig. 3 Results of a wear test

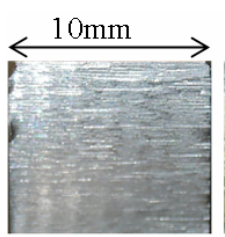

$N=0$

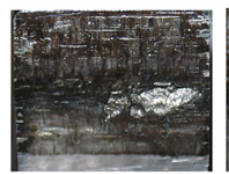

$N=4 \times 10^{5}$

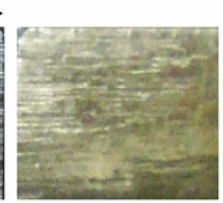

$N=1 \times 10^{5}$

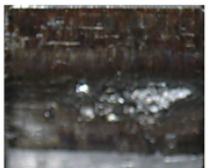

$N=6 \times 10^{5}$

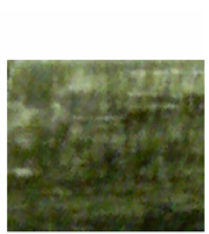

$N=2 \times 10^{5}$

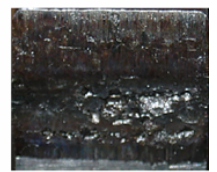

$N=1 \times 10^{6}$

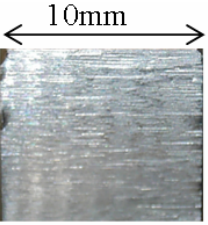

$N=0$

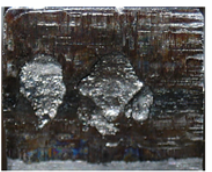

$N=4 \times 10^{5}$

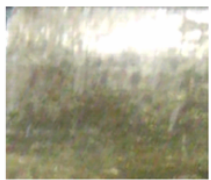

$N=1 \times 10^{5}$

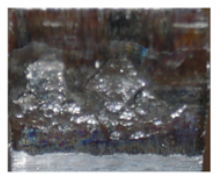

$N=6 \times 10^{5}$

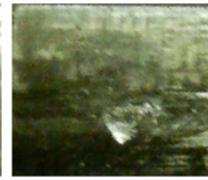

$N=2 \times 10^{5}$

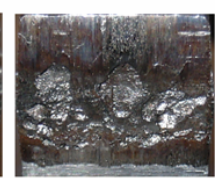

$N=1 \times 10^{6}$

(b) Tooth number 29

Fig. 4 Photographs of tooth surface of driving gear for several load repetition numbers $N$ (in cycles) 


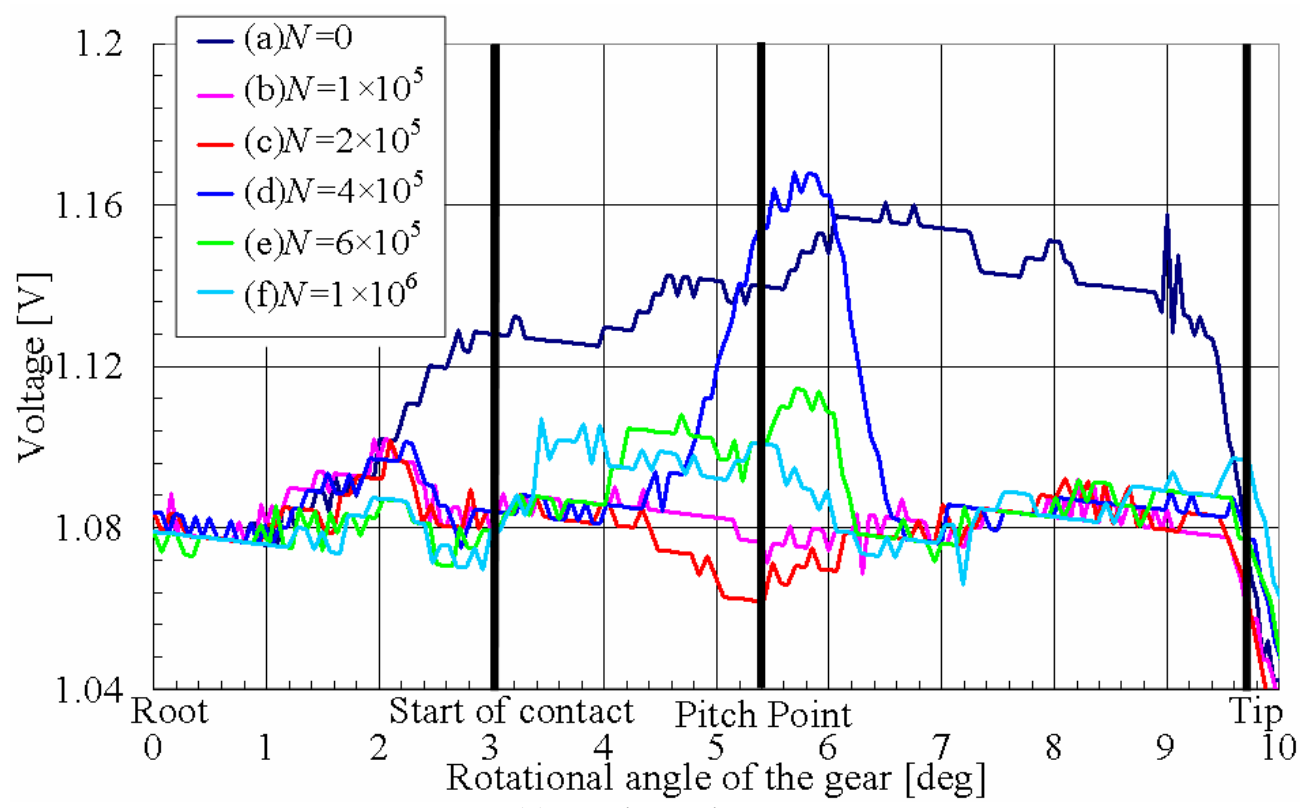

(a) Tooth number 15

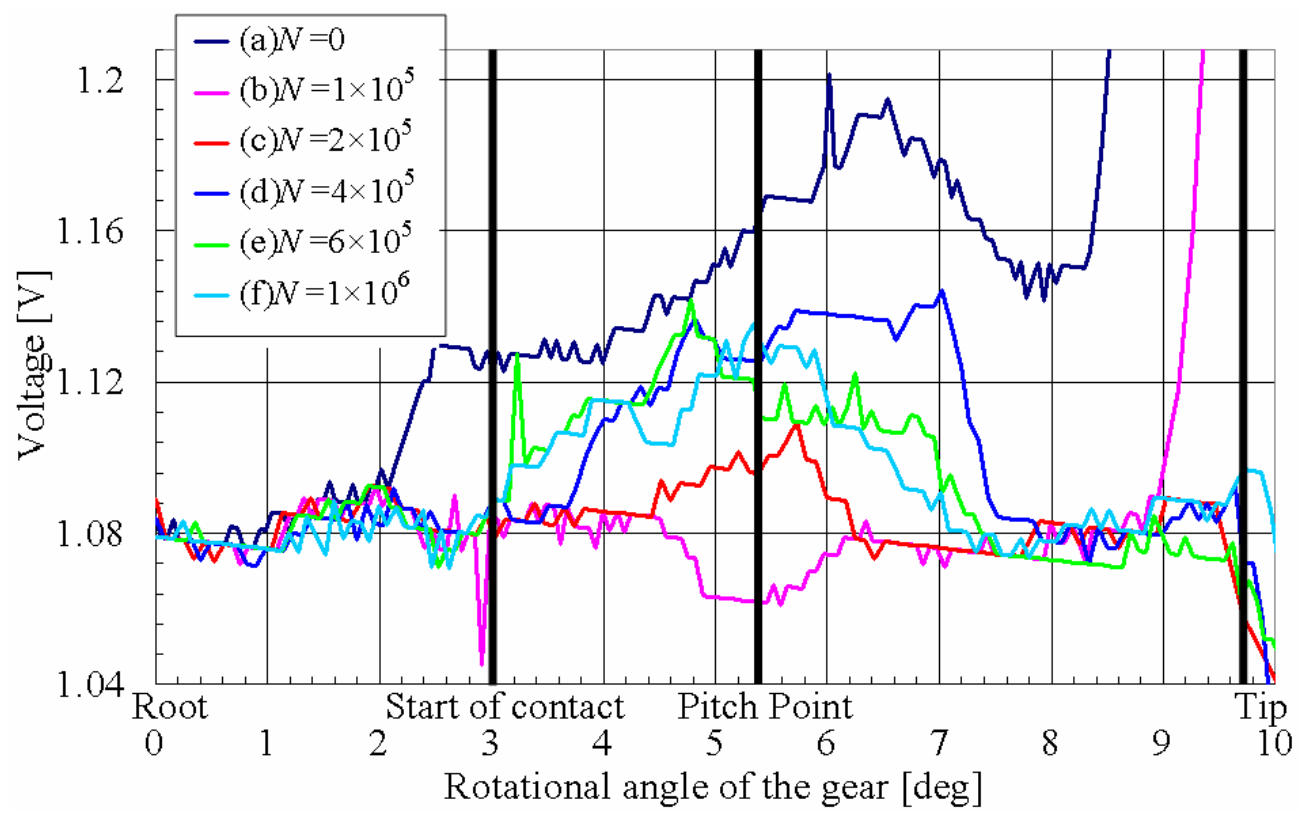

(b) Tooth number 29

Fig. 5 Voltage indicating laser scattering from driving-gear teeth surface as a function of the gear-rotation angle (compensated). Colors indicate the various load repetition numbers shown in the legend.

Furthermore, to properly diagnose damage, we present in Fig. 6 the difference between the laser-scatter signal for each load repetition number and the laser-scatter signal at $N=1 \times$ $10^{5}$ as a function of the driving-gear rotation angle, so that only the laser-scatter signal of the pitted area remains. Therefore, by calibrating the laser-scatter signal, we can diagnose the condition, location, and size of the pitting on the tooth surface. For example, from curve (f) - (b) in Fig. 6(a), the range of the increased laser-scatter signal was from 3.4 to 6.1 [deg]. Therefore, the calculated damage size was 2.69 [mm]. This agrees with the damage size measured by calipers, which gave 2.75 [mm] (the error for both measurements was approximately $3[\%]$ ). In the same way, from curve (f) - (b) in Fig. 6(b), the calculated damage size was $4.41[\mathrm{~mm}]$, which agrees with the measured size of 4.23 [mm] (the error of both measurements was approximately 4 [\%]). Therefore, we conclude that this method can 
be used to diagnose and verify the location and size of pitting to within 5 [\%].

As shown in Fig. 4(b), pitting initially occurred at tooth 29 at $N=2 \times 10^{5}$, which was shown by the laser-scattering results. In Figs. 7-9, we show, for the driving gear at $N=4 \times$ $10^{5}$, vibration data for the driving gear and the pedestal and adjacent-sound data, respectively. However, it is difficult to detect the damage on tooth 29 by analyzing these data. In comparison, the laser-scattering results shown in Fig. 6 reveals the occurrence of pitting much more rapidly and clearly. Therefore, we conclude that the proposed method can monitor tooth-surface conditions with sufficient accuracy to assess the lifetime of a gear.

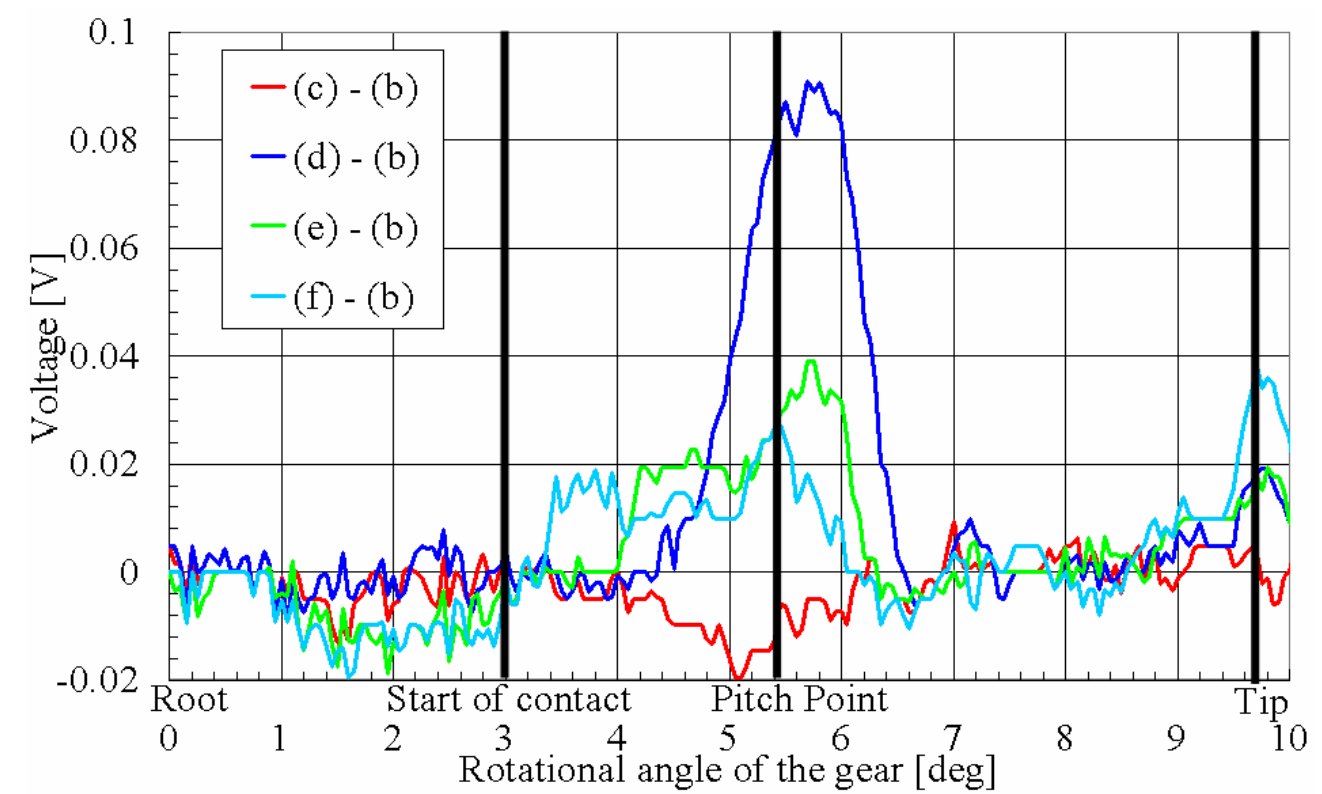

(a) Tooth number 15

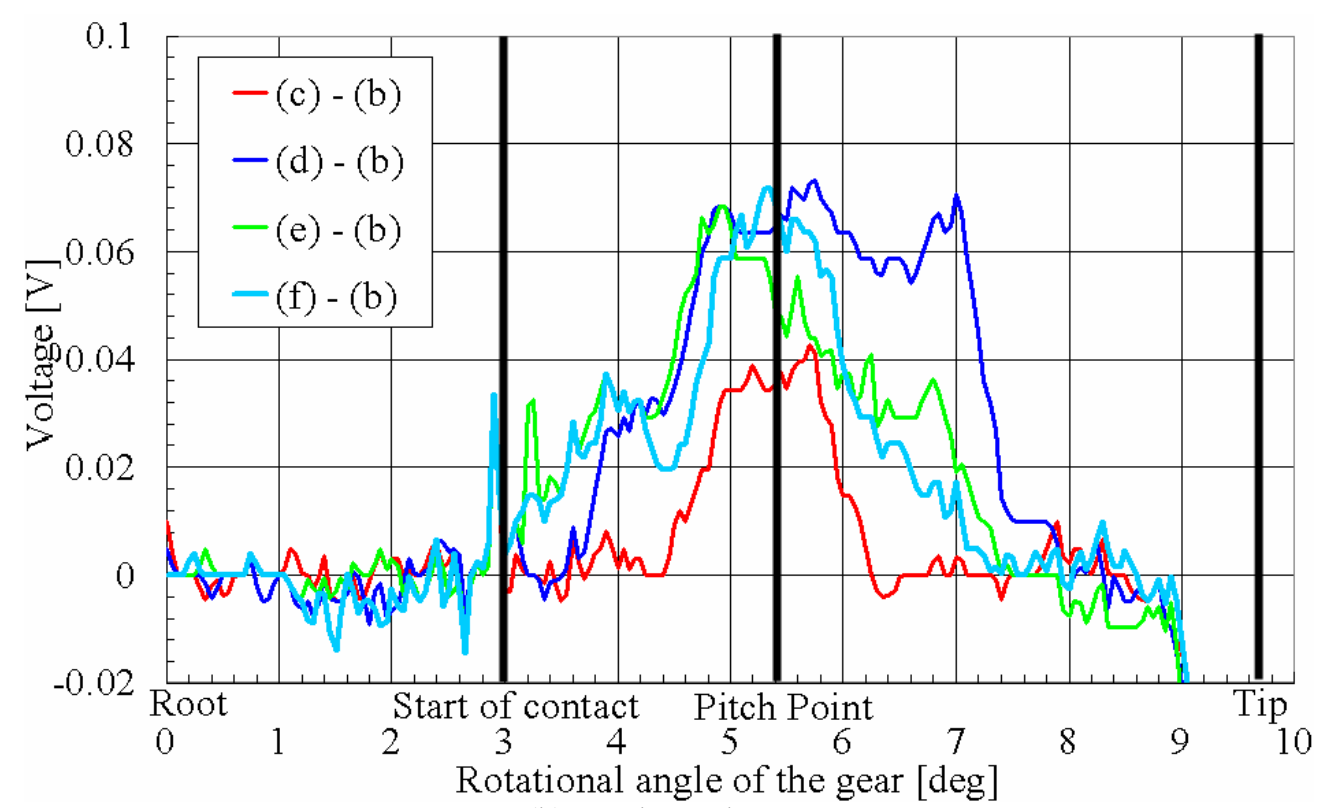

(b) Tooth number 29

Fig. 6 Difference between the laser scatterings at the load repetition numbers given in Fig. 5 and at $N=1 \times 10^{5}$ as a function of driving-gear rotation angle 


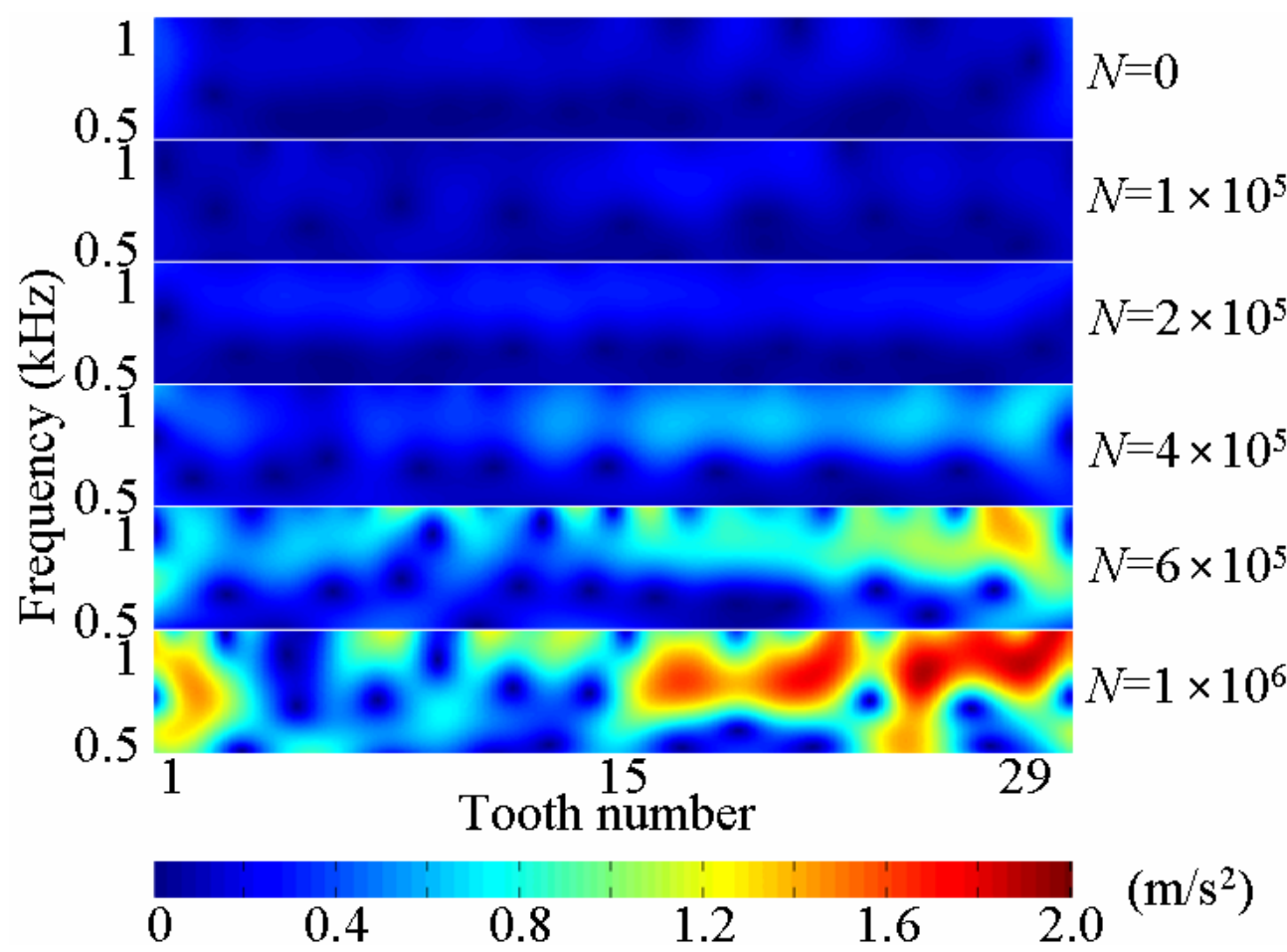

Fig. 7 Vibration of driving gear with continuous wavelet transform for each load repetition number

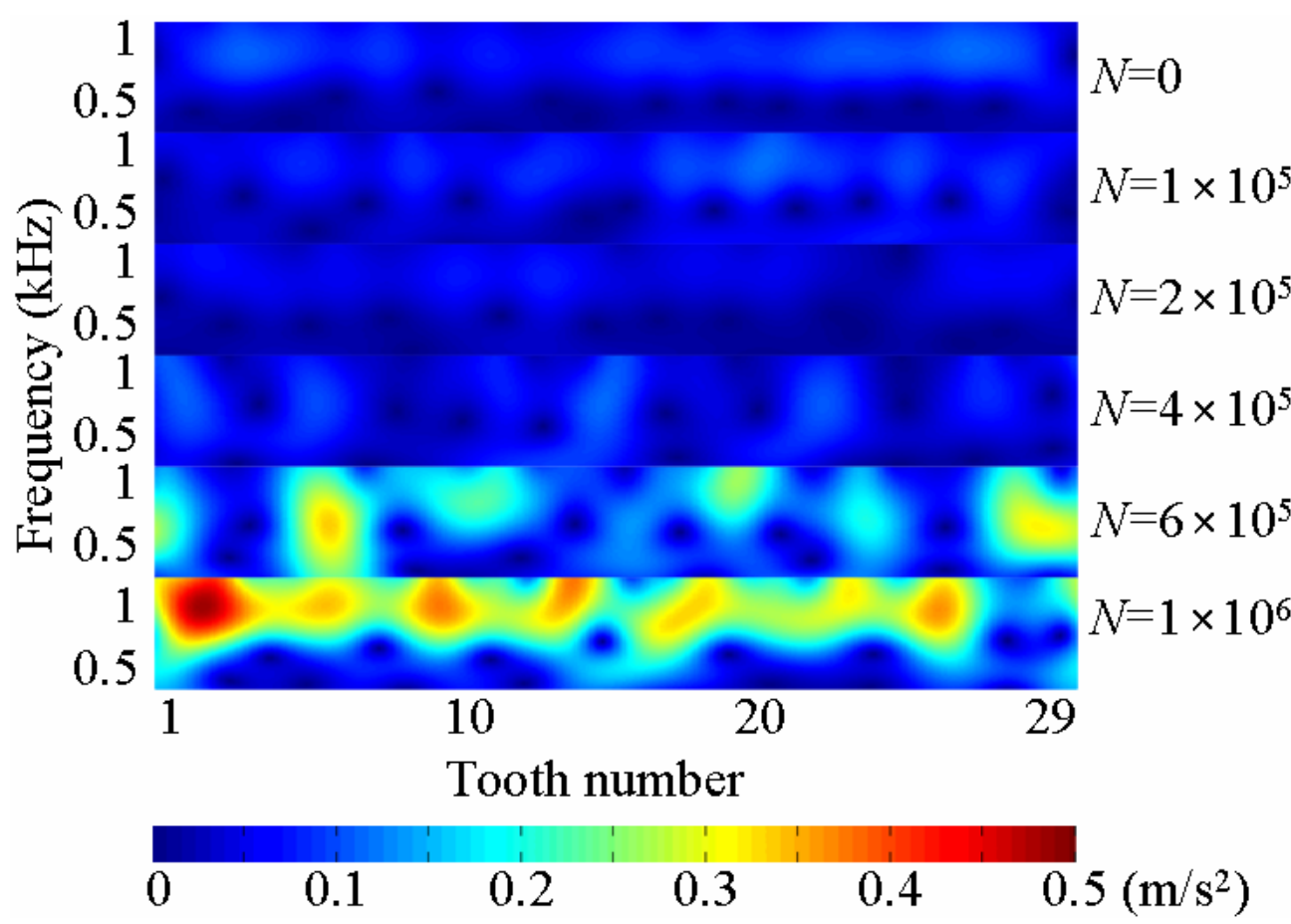

Fig. 8 Vibration of pedestal with continuous wavelet transform for each load repetition number 


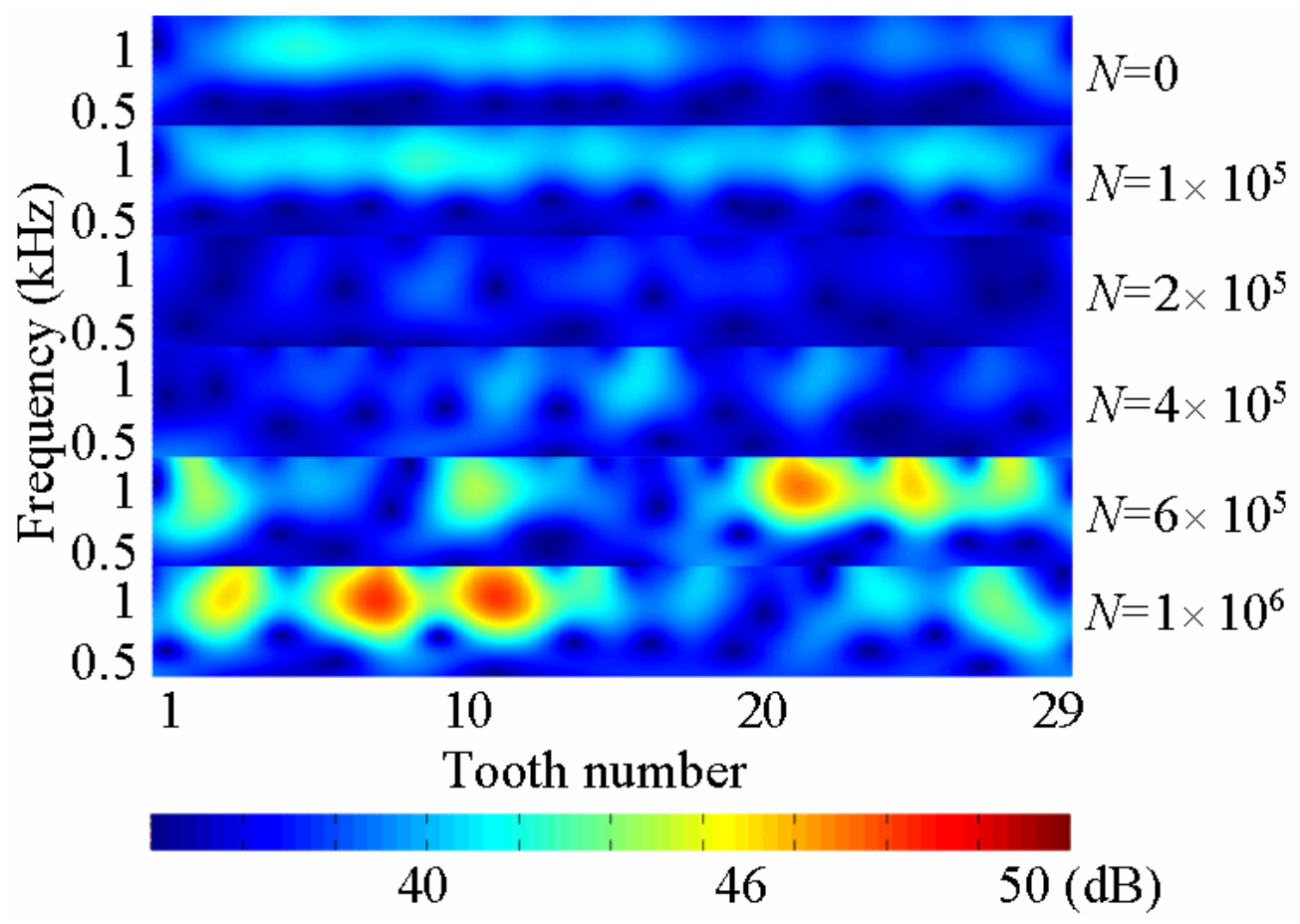

Fig. 9 Sound data from adjacent point with continuous wavelet transform for each load repetition number

\section{Measurements with Effects of Lubrication}

To use this method for practical purposes, a laser sensor was fixed in a gearbox, as shown in Fig. 10(a), to measure the laser scattered from the lubricated gear tooth. Below 60 [rpm], the apparatus could detect pitting. However, when the laser sensor became splattered with lubricant, because of the rotation of the gear, unreliable data were acquired. To prevent this, the laser beam was encased with square duct, as shown in Fig. 10(b). Both ends of the duct were sealed with a plastic board that was transparent at the laser wavelength. The use of this cover resulted in the acquisition of more repeatable data.

Furthermore, to find the best way to measure the laser scatter, we performed six experiments under various lubrication conditions (each of these conditions is given in Table 3). A nylon brush was positioned in the gearbox to brush away lubricant that accumulated on the surface of the rotation-gear teeth (see Fig. 11(a)). These experiments were performed with the gears lubricated either by an oil bath or by force-feed lubrication, as illustrated in Fig. 11. The gear testing apparatus and the test gear pair were the same as that used for the pitting experiment (after $N=1 \times 10^{6}$ ), because the purpose of this experiment was to find a way to accurately measure the tooth-surface damage. The input rotational speed was set at $60,120,180,240,360,480,600,720,840,960,1080,1320,1560$, and 1800 [rpm].

A sample of the data acquired is shown in Fig. 12. To clearly evaluate the range of damage (especially pitting), we include in this figure the data which were acquired under dry conditions (i.e., no lubrication) at 60 [rpm] (Red line). This figure shows data for three teeth. The large peaks correspond to scattering from the top tooth and the small peaks correspond to scattering from pitting. From Fig. 12, in the range below 360 [rpm], is hard to recognize the area of pitting due to pool of oil in the pitting spot and thick oil film on the tooth surface. However, as the rotational speed increases, the outline of the small peaks which show the pitting can be clearly recognized.

With this experiment, we evaluated the repeatability of the data and the accuracy of the apparatus. The repeatability of the data was evaluated from three trials, each of which measured laser scattering from the same tooth, and the repeatability was quantified as a 
function of the similarity of the wave forms. Explicitly, the repeatability was assigned four values: $0,33,66$, and 100 [\%]. The accuracy of the remote pitting length measurement was verified with a caliper, which yielded a pit length of $2.66[\mathrm{~mm}]$. The remotely measured pit length was calculated from the data with the help of Eq. (2), and the results are shown in Fig. 12. The accuracy was calculated with the following equation:

$$
\text { Accuracy }[\%]=\left(1-\left|x_{c^{-}} x_{m}\right| / x_{c}\right) \times 100 \text {. }
$$

where $x_{c}$ is the caliper-measured pit length of $2.66[\mathrm{~mm}]$, and $x_{m}$ is the value calculated from Eq. (2), which is based on the remotely acquired data.

Figures 13, 14, and 15 show the results for repeatability, accuracy, and total evaluation, respectively. By attaching the brush, the accuracy of pitting detection is improved for rotation speeds below 240 [rpm]. Under force-feed lubrication, pitting could be detected for every speed below 1800 [rpm]. As a result, we conclude that the proposed method can diagnose the pitting of teeth surface of lubricated gears in practical gearboxes.

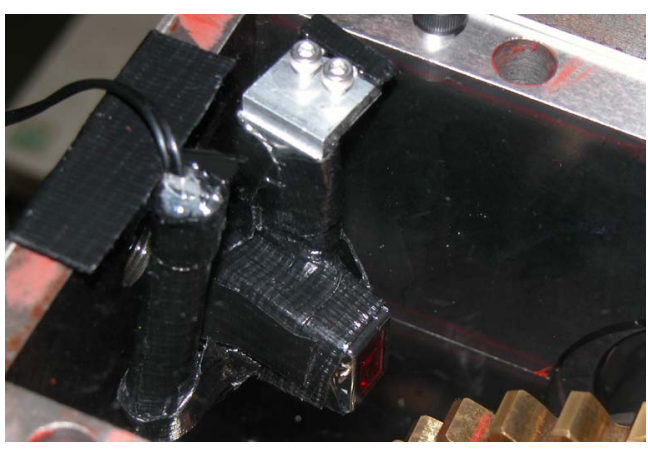

(a) Fixed laser sensor in the gearbox

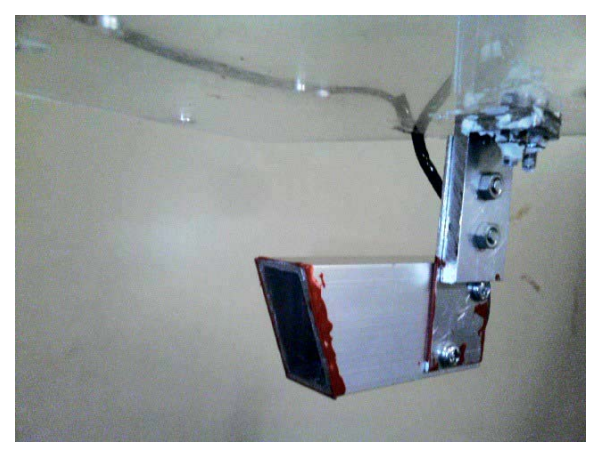

(b) A square pipe encased the laser beam

Fig. 10 Laser sensor in the gearbox

Table 3 Conditions of the experiment

\begin{tabular}{c|c|c|c}
\hline \hline Exp. No. & Oil temperature[K] & Lubrication method & Brush \\
\hline 1 & 279 & Oil bath & \\
2 & 279 & Oil bath & Attached \\
3 & 280 & Force feed & \\
4 & 313 & Oil bath & \\
5 & 313 & Oil bath & Attached \\
6 & 313 & Force feed & \\
\hline
\end{tabular}

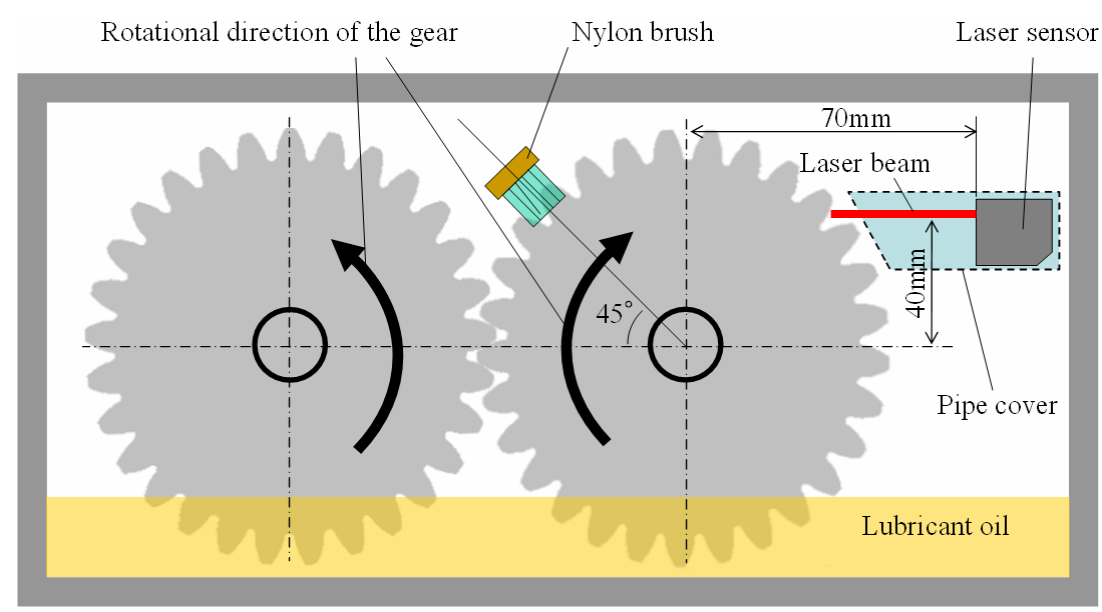

(a) Oil bath and Brush 


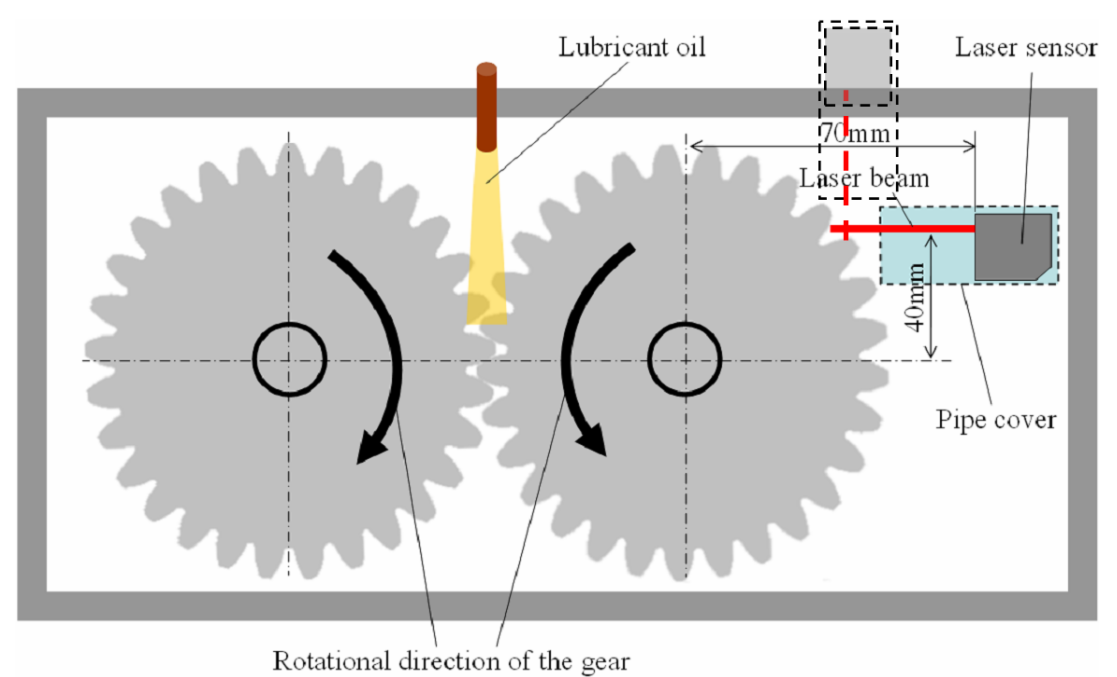

(b) Force feed

Fig. 11 Lubrication method and attached point of the brush

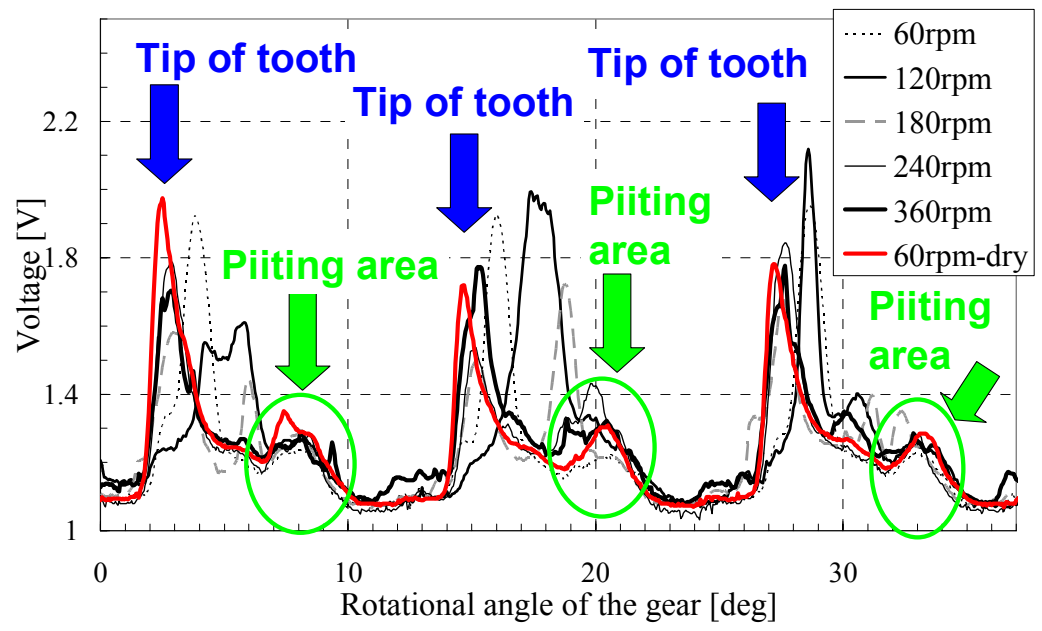

(a) $60-360 \mathrm{rpm}$

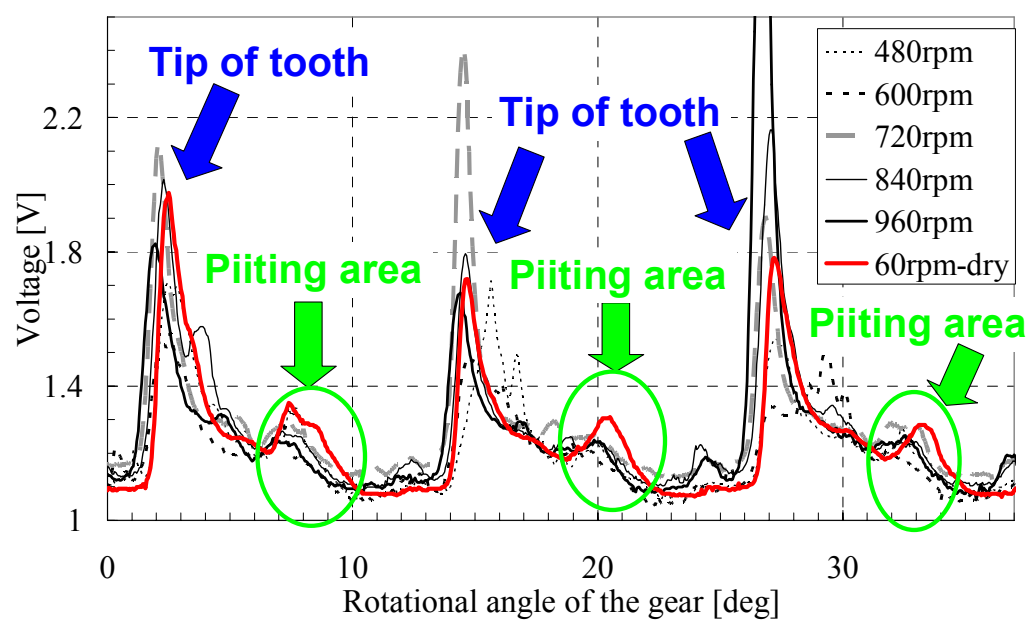

(b) $480-960 \mathrm{rpm}$ 


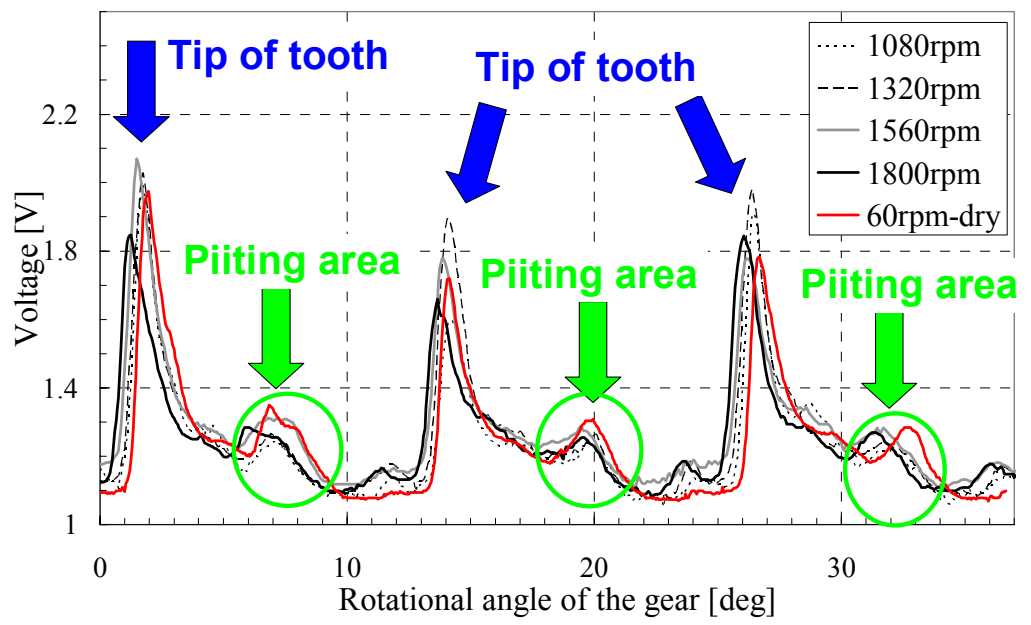

(c) $1080-1800 \mathrm{rpm}$

Fig. 12 Measured data of the laser reflection (Exp. No. 6)

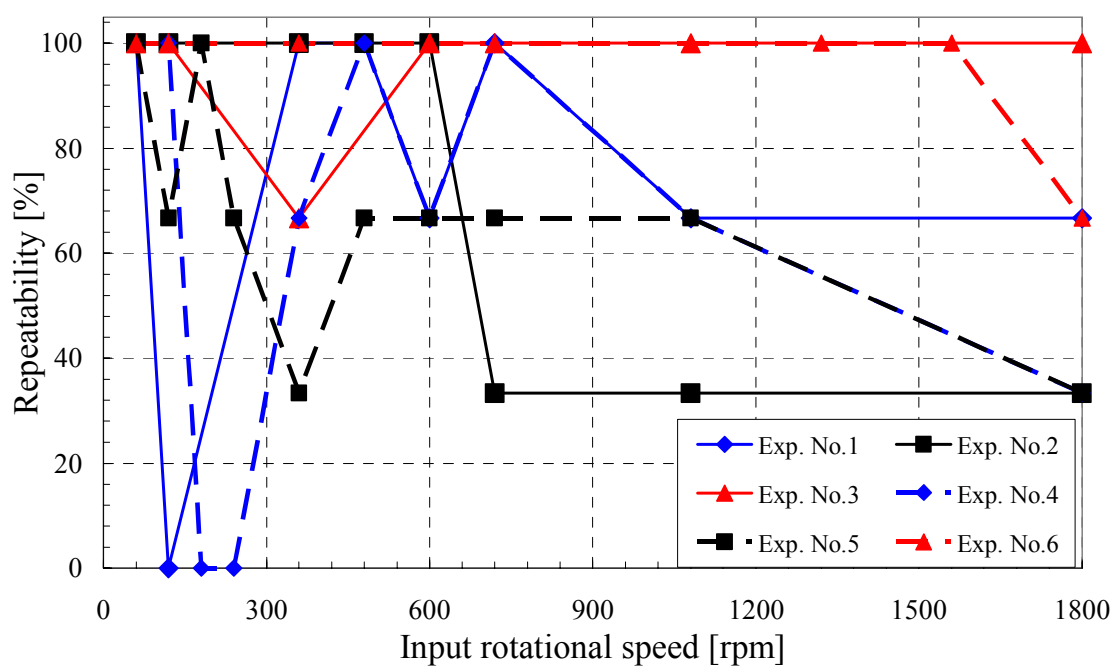

Fig. 13 Repeatability of laser-scattering data as a function of input rotational speed

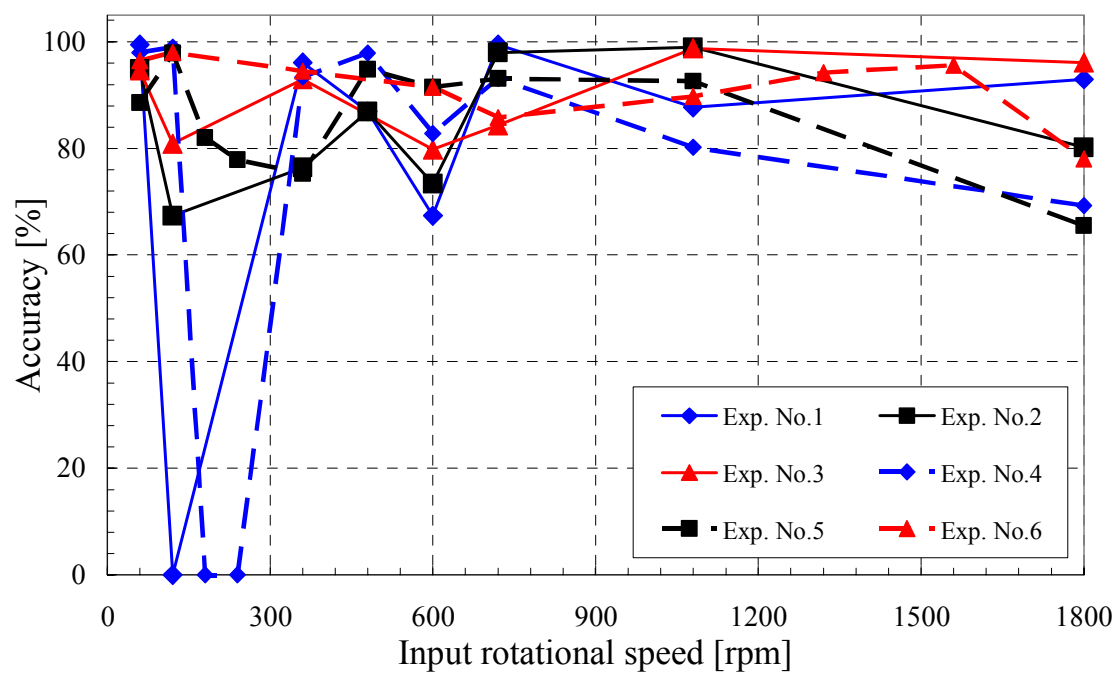

Fig. 14 Accuracy of the laser-scattering data as a function of input rotational speed 


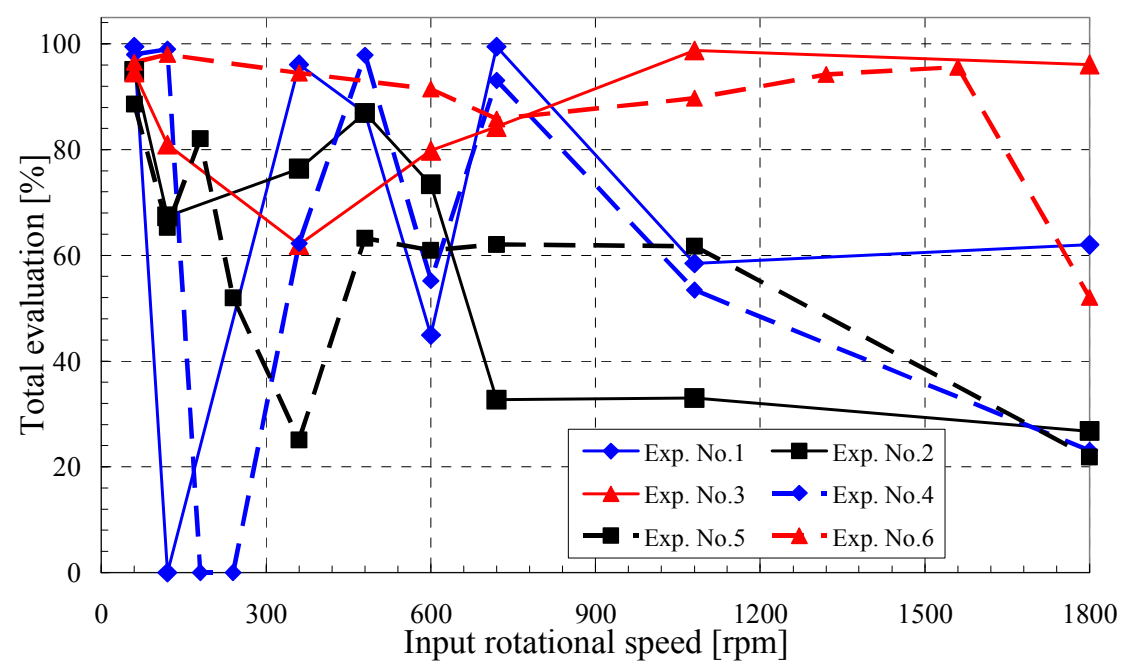

Fig. 15 Overall evaluations of laser-scattering data as a function of input rotational speed

\section{Computer-controlled damage diagnosis}

We have also developed an automatic damage diagnosis method with the ultimate goal being to create a system that can automatically diagnose gear damage. For example, such a system would be able to detect the number of damaged teeth, and from the remotely acquired data, calculate the length of the damaged area on the tooth surface. In this section, we discuss the method to automatically scan the length of the pitting area from the laser-scattering data.

Two thresholds were applied to the difference data of the laser-scatter signal (i.e., the voltage) between the damage data and the benchmark data. The benchmark data were defined as those with the lowest voltage, as described in section 4. Figure 16 shows the steps of this method as applied to curves (e) - (b) in Fig. 6(a). First, the first threshold was set at a relatively high voltage in the range $0.02-0.05$ [V] (Check 1 in Fig. 16) to find a damaged event. Second, the second threshold was set at 0.01 [V] (Check 2 in Fig. 16) because we estimated the noise amplitude of the un-pitted area to be 0.01 [V] from Fig. 6. If the curve exceeds the second-stage threshold over a range that includes where the curve exceeds the first-stage threshold, then the second-stage range is taken as the damaged area. Finally, to clarify both ends of this area, the points that include the data gradient angle are inverted and scanned along the data, as shown by Check 3 in Fig. 16. When the points are found, the damaged area is defined as the range between both the terminal points.

To confirm the validity of this method, we performed a pitting experiment, and compared the automatically detected pitting damage with that measured by calipers for each load repetition number. Dimensions of the test gear pair were the same as those given in Table 1. The same power-circulating-type gear testing machine was used as described in section 4, and the experimental conditions were the same as given in Table 2, except for the input torque that was set at $145[\mathrm{~N} \cdot \mathrm{m}]$.

Table 4 compares the results for pit size measured automatically in situ as described above with the results obtained with caliper measurements. The results given in Table 4(a) demonstrate that this method can detect all pitting events in dry conditions. The number of pitting events decreased upon increasing the first threshold voltage. However, when the first threshold voltage was 0.03 or 0.04 [V], damage was detected remotely by laser scanning in a place that was not damaged. We attribute this false positive to the large noise in the difference data. This result shows that for the proposed method to effectively diagnose gear-tooth damage, it is important to properly set the first threshold voltage and to smooth the difference data to accurately obtain remotely detected pit lengths. 


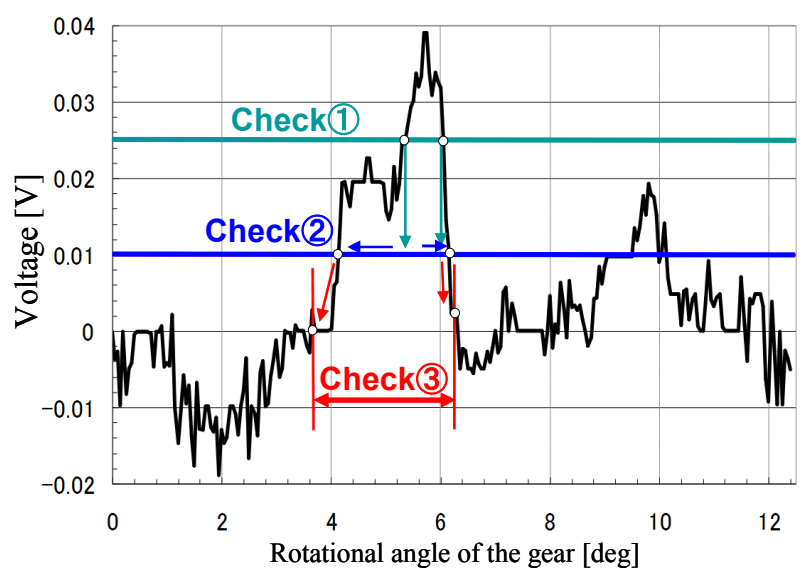

Fig. 16 Example of automatic damage diagnosis method. Method is applied to curve (e) (b) of Fig. 6(a).

Table 4 Comparison of pit size detected in situ by laser scanning with pit size measured with a caliper

(a) First (second) threshold $0.03(0.01)$ [V]

\begin{tabular}{c|c|c|c|c|c|c}
\hline \hline \multirow{2}{*}{$\begin{array}{c}\text { Load } \\
\text { repetition } \\
\text { number }\end{array}$} & \multicolumn{5}{|c}{ Damage size [mm] } \\
\cline { 2 - 7 }$N\left[\times 10^{\wedge} 5\right]$ & \multirow{2}{*}{$\begin{array}{c}\text { Measured } \\
\text { with a caliper }\end{array}$} & \multicolumn{5}{|c}{ Calculated with a computer } \\
\cline { 3 - 7 } & & $60[\mathrm{rpm}]$ & $60[\mathrm{rpm}]$ & $600[\mathrm{rpm}]$ & $1200[\mathrm{rpm}]$ & $1800[\mathrm{rpm}]$ \\
\hline 4 & 0.00 & & 0.88 & & & \\
6 & 0.00 & & & & 1.13 & \\
10 & 0.88 & 0.77 & 1.38 & & 1.75 & \\
20 & 1.34 & 0.85 & 3.93 & & 0.34 & 0.36 \\
50 & 1.63 & 1.87 & 1.75 & 2.29 & 1.83 & 2.00 \\
100 & 2.40 & 5.70 & 4.84 & 3.93 & 4.32 & 4.84 \\
\hline
\end{tabular}

(b) First (second) threshold: 0.04 (0.01) [V]

\begin{tabular}{c|c|c|c|c|c|c}
\hline \hline \multirow{2}{*}{$\begin{array}{c}\text { Load } \\
\text { repetition } \\
\text { number }\end{array}$} & \multicolumn{5}{|c}{ Damage size [mm] } \\
\cline { 3 - 7 }$N\left[\times 10^{\wedge} 5\right]$ & \multirow{2}{*}{$\begin{array}{c}\text { Measured } \\
\text { with a caliper }\end{array}$} & \multicolumn{5}{|c}{ Calculated with a computer } \\
\cline { 3 - 7 } & & $60[\mathrm{rpm}]$ & $60[\mathrm{rpm}]$ & $600[\mathrm{rpm}]$ & $1200[\mathrm{rpm}]$ & $1800[\mathrm{rpm}]$ \\
\hline 4 & 0.00 & & 0.88 & & & \\
6 & 0.00 & & & & & \\
10 & 0.88 & 0.77 & 1.34 & & & \\
20 & 1.34 & & 3.93 & & & \\
50 & 1.63 & 1.87 & 1.75 & 2.29 & 1.26 & 2.00 \\
100 & 2.40 & 5.70 & 4.85 & 3.93 & 4.32 & 4.84 \\
\hline
\end{tabular}

(c) First (second) threshold: 0.05 (0.01) [V]

\begin{tabular}{c|c|c|c|c|c|c}
\hline \hline \multirow{2}{*}{$\begin{array}{c}\text { Load } \\
\text { repetition } \\
\text { number }\end{array}$} & \multicolumn{5}{|c}{ Damage size [mm] } \\
\cline { 3 - 7 }$N\left[\times 10^{\wedge} 5\right]$ & \multirow{2}{*}{$\begin{array}{c}\text { Measured } \\
\text { with a caliper }\end{array}$} & \multicolumn{5}{|c}{ Calculated with a computer } \\
\cline { 3 - 7 } & & Dry & \multicolumn{4}{|c}{ Force feed lublication } \\
\hline 4 & 0.00 & & & & & \\
6 & 0.00 & & & & & \\
10 & 0.88 & & 1.34 & & & \\
20 & 1.34 & & 3.93 & & & \\
50 & 1.63 & 1.87 & 1.75 & 2.29 & 1.05 & 2.00 \\
100 & 2.40 & 5.72 & 5.50 & 3.93 & 4.24 & 5.70 \\
\hline
\end{tabular}




\section{Conclusions}

We developed a new gear-tooth diagnosis method that uses laser-beam scattering from the gear teeth to remotely detect gear-tooth damage. First, the tooth surface was irradiated by a zone-covering laser beam from an oblique direction, and the irradiated zone was scanned along the tooth's surface by the rotation of the gear. The variations of laser scattering between the initial and actual conditions were compared and used to estimate the condition of the tooth surface.

To use this method for practical purposes, a laser sensor was fixed in a gearbox, and laser scattering from the lubricated gear was measured. In the speed range below 60 [rpm], pitting could be detected from the measured data. However, when the laser sensor was splattered by lubricant from the rotation gear, the data became unreliable. To prevent this condition, a cover was made with a square duct to encase the laser beam. Furthermore, a brush was positioned in the gearbox to brush away lubricant that was accumulated on the tooth surface of the gear. These experiments were performed both under conditions of an oil bath lubrication and force-feed lubrication. With the cover in place, the data became more reproducible. By attaching the brush, the accuracy of pitting detection was improved in the speed range below 240 [rpm]. For force-feed lubrication, pitting could be detected for all speed ranges up to $1800[\mathrm{rpm}]$. Therefore, we conclude that the proposed method can diagnose the tooth surface of a lubricated gear in a practical gearbox.

Finally, we developed an algorithm to determine the length of a pit from the laser-scattering data. Under dry conditions, this method could detect all occurrences of pitting. To improve the accuracy with which the method determines pit length, it is important to properly define the first threshold voltage and to smooth the difference data.

\section{Acknowledgements}

We gratefully acknowledge Tatshurou Sugiyama, Kenta Imaoka, Takeshi Sakamoto, and Yusuke Inoue for carrying out the experiments and the analyses in this article.

\section{References}

(1) Oda, S., et al., Acoustic Emission in Bending Fatigue Process of Spur Gear Teeth, Transactions of the Japan Society of Mechanical Engineers, Series C, Vol.58, No. 551, (1992), pp. 2219-2225, (in Japanese).

(2) Umezawa, K., et al. Trial of Prognosis of Failure with Gear sound, Transactions of the Japan Society of Mechanical Engineers, Series C, Vol.49, No. 443, (1983), pp. 1210-1216, (in Japanese).

(3) Ohue, Y. and Yoshida, A., Failure Diagnosis on Tooth Surface of Gears by Continuous and Discrete Wavelet, Journal of Japanese Society of Tribologists, Vol. 48-No. 10, (2003), pp. 828-835, (in Japanese).

(4) F. K. Choy, et al., Damage Identification of a Gear Transmission using Vibration Signatures, Proc. of the JSME Int. Conf. on Motion and Power Transmissions (MPT-2001), Fukuoka, Japan, JSME (2001), pp. 84-89.

(5) Tanaka, E. et al., The Damage Diagnosis of Gear Tooth Surface by Using a Laser Beam Reflection, Proc. of the Int. Conf. on Mechanical Transmissions (ICMT2006), Chongqing, China, (2006), pp. 990-995.

(6) Tanaka, E. et al., The Damage Diagnosis of Gear Tooth Surface by Using a Laser Beam (Study toward the Practical Use under the Condition of Lubricant), Proc. of the 
JSME-KSME Joint Int. Conf. on Manufacturing, Machine Design and Tribology (ICMDT2007), Hokkaido, Japan, (2007), CD-ROM. 